\title{
Нелинейная по концентрации поверхностных состояний модель барьера Шоттки и расчет вольт-амперных характеристик диодов на основе SiC и его твердых растворов в составной модели токопереноса
}

\author{
() В.И. Алтухов ${ }^{1}$, А.В. Санкин ${ }^{1}$, А.С. Сигов ${ }^{2}$, Д.К. Сысоев ${ }^{1}$, \\ Э.Г. Янукян ${ }^{1}$, С.В. Филиппова ${ }^{3}$ \\ ${ }^{1}$ Северо-Кавказский фредеральный университет, \\ 355009 Ставрополь, Россия \\ ${ }^{2}$ Московский институт радиоэлектроники и автоматики, \\ 119454 Москва, Россия \\ ${ }^{3}$ Пятигорский медико-фрармацевтический институт, \\ 357500 Пятигорск, Россия \\ E-mail: altukhov@mail.ru
}

(Получена 10 апреля 2017 г. Принята к печати 22 апреля 2017 г.)

\begin{abstract}
Предложена модифицированная нелинейная по концентрации поверхностных состояний модель барьера Шоттки, содержащая локальный квазиуровень Ферми на границе раздела, обусловленного избыточным поверхностным зарядом. Такой подход позволяет объяснить наблюдающееся подобие вольт-амперная характеристика диодов с барьером Шоттки $\mathrm{M} /(\mathrm{SiC})_{1-x}(\mathrm{AlN})_{x}$ и гетеропереходов на основе $\mathrm{SiC}$ и его твердых растворов с учетом $\Phi_{g} \approx \Phi_{\text {В }}$ Результаты расчетов высоты барьеров Шоттки согласуются с данными опытов, полученными по фототоку для металлов (M): Al, Ti, Cr, Ni. Вольт-амперные характеристики в составной аддитивной модели токопереноса согласуются с данными экспериментов для систем $n-\mathrm{M} / p-(\mathrm{SiC})_{1-x}(\mathrm{AlN})_{x}$ и $n-6 H-\mathrm{SiC} / p-(\mathrm{SiC})_{0.85}(\mathrm{AlN})_{0.15}$.
\end{abstract}

DOI: 10.21883/FTP.2018.03.45623.8587

\section{1. Введение}

Вопрос формирования и вычисления высоты потенциального барьера в гетеропереходах $\Phi_{g}$ (высота барьера в $p-n$-переходе) и барьера Шоттки $\Phi_{\mathrm{B}}$ на контакте металл-полупроводник является актуальной задачей теории полупроводников [1-6]. Одним из важнейших вопросов до сих пор остается проблема построения обобщенной модели токопереноса через гетеропереход (ГП) типа $n-\mathrm{SiC} / p-(\mathrm{SiC})_{1-x}(\mathrm{AlN})_{x}$ или через контакт металл-полупроводник с барьером Шоттки $\left(\right.$ БШ-M/SiC $\left.{ }_{1-x}(\mathrm{AlN})_{x}\right)$. В реальных ГП и контактах с БШ существует целый каскад механизмов токопереноса, часто одновременно действующих в разных, а иногда и в одном и том же интервале приложенных внешних напряжений $U[3,4]$. Поэтому ни одна из известных моделей (см. [4]) не дает возможности до конца правильно интерпретировать экспериментальные данные, в том числе зависимость $I(U)$ (BAX) от плотности поверхностных состояний вблизи границы раздела, от температуры, от состава $x$ твердого раствора $(\mathrm{SiC})_{1-x}(\mathrm{AlN})_{x}$.

В настоящей работе приведен расчет высоты потенциального барьера в области гетероперехода и ВАХ с резонансным квазиуровнем Ферми $E_{\mathrm{F}}$; с единых позиций, в рамках предложенной нами составной-аддитивной модели, объясняются особенности и сходство поведения $\mathrm{BAX}$ гетероструктур - диодов типа $\mathrm{M} / \mathrm{SiC}_{1-x}(\mathrm{AlN})_{x}$ и $n-\mathrm{SiC} / p$ - $(\mathrm{SiC})_{1-x}(\mathrm{AlN})_{x}$. Составная-аддитивная по видам токопереноса модель представляет собой справедливую в широкой области напряжений сумму токов, связанную с тремя известными механизмами токопереноса. Это термоэлектронная, полевая и термополевая эмиссии (см. [3] с. 91 формула (1.106), с. $96-(1.109)$, c. $97-(1.111))$.

\section{2. Модифицированная модель барьера Шоттки}

Рассмотрим модель барьера Шоттки с локализованными в области контакта состояниями дефектов (БШ [1-3]), но в более высоком (нелинейном) по концентрации дефектов $\left(N_{i}=c \cdot 10^{13} \mathrm{~cm}^{-2} \cdot{ } \mathrm{B}^{-1}, c=0-30\right.$, где $c$ - удобный параметр - „концентрация“ ${ }^{6}$ в единицах $\left.10^{13} \mathrm{~cm}^{-2} \cdot{ }^{-1}\right)$ приближении. В этом подходе, наряду с концентрацией дефектов $N_{i}$, вводятся числа заполнения $n_{x}(c)$ локализованных на границе раздела поверхностных состояний. Эти состояния определяются видом гамильтониана системы. Такой подход дает более высокие значения барьера Шоттки и при малых $N_{i}$ $\left(N_{i}<10^{13} \mathrm{~cm}^{-2} \cdot\right.$ эB $\left.^{-1}\right)$ ведет к лучшему (на $15-20 \%$ ), чем классическая формула Бардина, Шоттки-Мотта, согласию с данными опытов. В предлагаемой модели высота барьера $\Phi_{\mathrm{B}}^{x}$ определяется формулой $[6,7]$

$$
\Phi_{\mathrm{B}}^{x}(c)=p+k \eta c 2 n_{x}(c)
$$




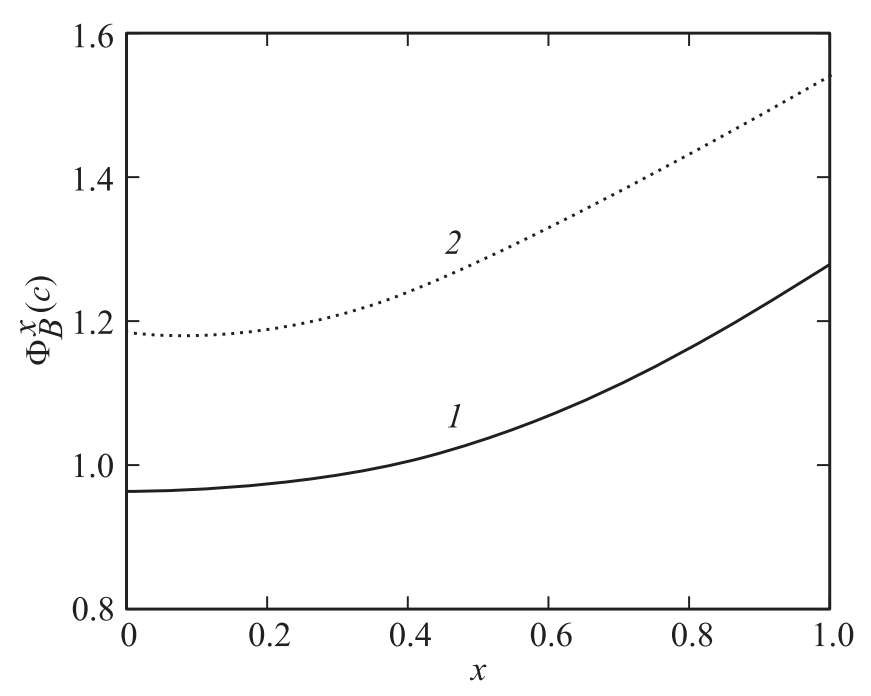

Рис. 1. Высота барьера Шоттки как функция состава $x$ для модельной системы $n-\mathrm{Al} / p-(\mathrm{SiC})_{1-x}(\mathrm{AlN})_{x}$ при $c=4 ; E_{1}=0.3 E_{g}$ (кривая 1) и $E_{2}=0.5 E_{g}$ (кривая 2).

При этом для чисел заполнения локализованных состояний $n_{x}(c)$ получаем:

$$
\begin{gathered}
n_{x}(c)=(1 / \pi) \operatorname{arccot} \delta_{x}(c), \\
\Gamma \delta_{x}(c)=p-\left(1-\xi_{i}\right) E_{g}^{x}+k \eta c(1-c v) .
\end{gathered}
$$

Здесь численный множитель $k=k(\lambda=3 \AA)=0.272$ эВ [7], $p=\Phi_{m}-\chi, \Phi_{m}-$ работа выхода электрона из металла, $\chi$ - сродство к электрону; энергия поверхностных состояний $E_{i}=E_{g}^{x} \xi_{i}, \xi_{i}=0.3(0.5 ; 0.7), \Gamma-$ ширина состояния $E_{i}, E_{g}^{x}$ - ширина запрещенной зоны. Параметр ширины двойного слоя $\lambda=3 \eta \AA$ (см. [1], с. 597). Значение $\lambda=3 \AA-$ оценка для контактов металл-карбид кремния $[1] ; \eta=0.5-2.0-$ учет возможного изменения $\lambda$ в системах $\mathrm{M} /(\mathrm{SiC})_{1-x}(\mathrm{AlN})_{x}$. При $E_{i}=E_{g}^{x} / 2=E_{\mathrm{F}}$ имеем $\delta_{x}(c)=\left(E_{i}-E_{\mathrm{F}}\right) / \Gamma=0$ и $n_{x}(c) \approx n_{0}=1 / 2$. В приближении $c^{2}$ для барьера Шоттки $\Phi_{\mathrm{B}}^{x}(c)$ имеем: $2 n_{x}(c) \approx 1-c v$ $(c v \leq 1)$, где $v=0-0.033$ - коэффициент разложения $n_{x}(c)$ по $c$ в реальном интервале концентраций $c=0-30$ (см. [6,7]). При этом для $Г \delta_{x}(c)$ получаем (3) и для барьера Шоттки $\Phi_{\mathrm{B}}^{x}(c)$ формулу (1). В первом порядке по $c$, согласно $(1)-(3)$, получаем формулу для высоты барьера Шоттки по модели Давыдова-Лебедева-Тихонова [1].

Результаты расчетов высоты барьера Шоттки представлены на рис. 1 как функции состава $x$. По значениям фототока структур $\mathrm{M} /(\mathrm{SiC})_{0.4}(\mathrm{AlN})_{0.6}$ на опыте была определена высота барьеров Шоттки для металлов (M): $\mathrm{Al}$, Ti, Cr, Ni: 1.78, 1.85, 1.98, 2.16 эВ [8]. Эти значения согласуются с данными расчетов по модифицированной модели барьера Шоттки $\Phi_{\mathrm{B}}^{x}(c): 1.74,1.90,1.78$, 2.24 эВ $[6,7]$ при концентрациях $N_{i}=6 \cdot 10^{13} \mathrm{~cm}^{-2} \cdot$ эВ $^{-1}$ $(c=6)$. Значения для $\mathrm{Cr}$ выпадают из общего ряда по причине использованного в расчете известного значения его работы выхода [1].

\section{3. Вольт-амперные характеристики диодов на основе карбида кремния}

В гетеропереходах типа $n-\mathrm{SiC} / p-(\mathrm{SiC})_{1-x}(\mathrm{AlN})_{x}$ на границе перехода в области с высокой плотностью состояний носителей заряда появляется резонансный квазиуровень $E_{\mathrm{F}}$. При этом носители тока проникают через барьер эффективно, как в металл, и токопрохождение через $\Phi_{g}^{x}$ будет носить характер термоэлектронной $(t e)$, полевой $(p)$ или термополевой $(t p)$ эмиссии в зависимости от напряжения $U$. Этим, как было отмечено, объясняется качественное сходство ВАХ диодов с барьером Шоттки и обычных $p-n$-переходов [3]. $\mathrm{B}$ таких случаях для описания ВАХ диодов на основе $\mathrm{SiC}$ в широкой области напряжений $(0<U<5-15 \mathrm{~B})$ нами предложено использовать составную-аддитивную по токам модель. В этой модели в области низких напряжений $(0<U<0.5 \mathrm{~B})$ составляющая тока определяется термоэлектронной эмиссией. Составляющая полевой эмиссии проявляется в области напряжений $0.5<U<2.5$ В. Термополевая эмиссия наблюдается в области напряжений $2.4<U<5 \mathrm{~B}[3-5]$.

В области низких напряжений $(0<U<0.5$ В $)$ составляющая тока термоэлектронной эмиссии будет иметь вид [3]

$$
\begin{gathered}
I_{t e}(U, x)=I_{s s}^{x}\left[\exp \left(\frac{q U}{n k T}\right)-1\right], \\
I_{s s}^{x}=s A^{*} T_{i}^{2} \exp \left(-\frac{\Phi_{\mathrm{B}}^{x}(c)}{m k T}\right),
\end{gathered}
$$

где $I_{s s}^{x}$ - предэкспоненциальный множитель, $s-$ площадь контакта (порядка 2-5 мм $\left.{ }^{2}\right), x-$ состав, $A^{*}-$ постоянная Ричардсона, равная $120\left(m_{e}^{*} / m_{e}\right) \mathrm{A} / \mathrm{cm}^{2} \cdot \mathrm{K}^{2}$; $q$ - электронный заряд, $n$ и $m$-- факторы идеальности.

Составляющую полевой эмиссии с учетом опытных данных [4,5], проявляющуюся в области напряжений $0.5<U<2.5 \mathrm{~B}$, можно представить в виде [3-5]

$$
\begin{gathered}
I_{p}(U, x)=I_{p}^{x} \exp \left(\frac{q U}{n_{1} E_{00}^{x}}\right), \\
I_{p}^{x}=s A_{i}^{* *} T_{i}^{2} \exp \left(-\frac{\Phi_{\mathrm{B}}^{x}(c)}{E_{00}^{*}}\right) .
\end{gathered}
$$

Здесь $n_{1}-$ фактор идеальности для $I_{p}(U, x) ; A_{i}^{* *}-$ коэффициент, связанный с постоянной Ричардсона $A^{*}$, сложно зависящий от параметров соответствующего исходного материала [3]; $E_{00}^{*}=m_{1}^{i} E_{00} \quad\left(E_{00} \approx 20\right.$ мэВ $)$. Фактор идеальности $m_{1}^{i}$ и характеристическую энергию заданного материала $E_{00}$ можно определить по данным опытов. 


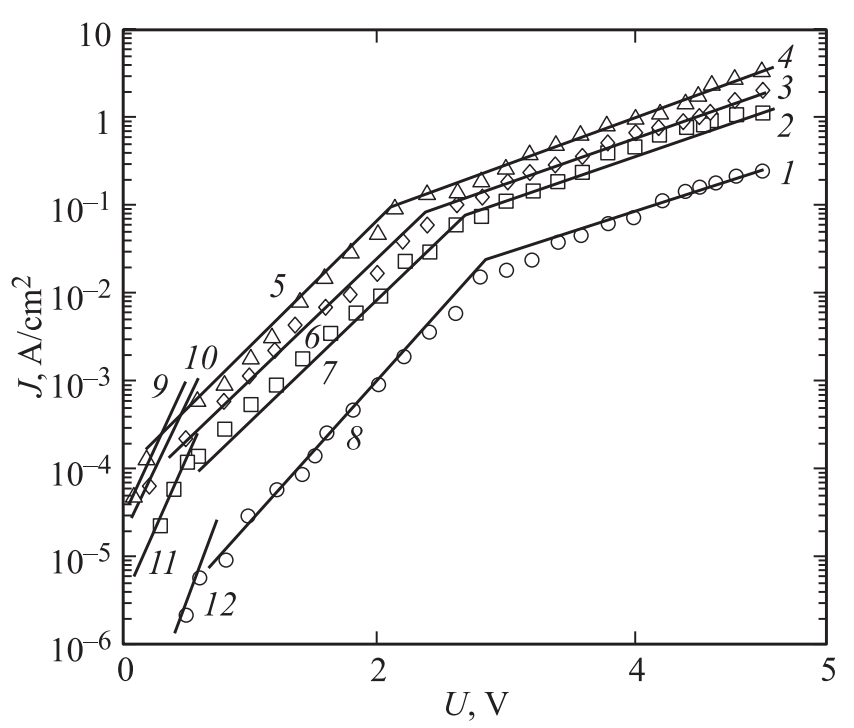

Рис. 2. Зависимость ВАХ $-J(U)$ для гетеропереходов $n-6 H-\mathrm{SiC} / p-(\mathrm{SiC})_{0.85}(\mathrm{AlN})_{0.15}$ при температурах $T_{i}=77,273$, $361,418 \mathrm{~K}$ : точки - эксперимент $[4,5,8]$; прямые линии 1 12 - данные расчетов при $\Phi_{\mathrm{B}}=1.7$ э.

Для термополевой эмиссии в области $2.5<U<5$ В напряжений согласно $[3,4]$ получаем:

$$
\begin{aligned}
& I_{t p}(U, x)=I_{t p}^{x} \exp \left(\frac{q U}{n_{2} E_{0}^{x}}\right), \\
& I_{t p}^{x}=s B_{i} T_{i}^{2} \exp \left(-\frac{\Phi_{\mathrm{B}}^{x}(c)}{E_{0}^{*}}\right),
\end{aligned}
$$

где $n_{2}$ и $m_{2}^{i}$ - фактор идеальности для $I_{t p}(U, x)$; $E_{0}^{*}=m_{2}^{i} E_{0}, E_{0}=E_{00} \operatorname{cth}\left(E_{00} / k T\right) ; c$ - концентрация поверхностных дефектов; $i=1,2,3,4$ и $E_{0}$ - характеристическая энергия материала. Зависимость $T_{i}^{2}$ в (5) и (6) определена исходя из данных опытов $[4,5]$.

Результаты расчетов по формулам (4)-(6) представлены на рис. 2: прямые линии 1-12 представляют собой расчетные значения ВАХ модели. Фигурными точками обозначены соответствующие экспериментальные данные. Значения параметров идеальности составной-аддитивной модели - $n, m ; n_{1}, m_{1}^{i}, n_{2}, m_{2}^{i}$ приведены далее.

На рис. 2 представлены ВАХ для термоэлектронной $(s s)$, полевой $(p)$ и термополевой эмиссии $(t p)$ при температурах: $1-77,2-273,3-361,4-418 \mathrm{~K}$. Прямые $1-4$ - термополевая эмиссия $(t p) ; 5-8-$ полевая эмиссия $(p) ; 9-12$ - термоэлектронная эмиссия $(s s)$. Согласно [3], $I_{t p}^{x}$ - предэкспоненциальный множитель термополевого тока. В расчете для этого множителя использованы значения: $I_{t p}^{x}=1,3.3,5,7\left(10^{-3} \mathrm{~A} / \mathrm{cm}^{2}\right)$, соответствуюшие прямым 1, 2, 3, 4 на рис. 2. При $T_{i}=77$, $273,361,418 \mathrm{~K}$ фактор идеальности термополевой эмиссии $(t p)$ около $q U[3]$ составляет $n_{2}=5$, а фактор идеальности около $\Phi_{\mathrm{B}}$ составляет: $m_{2}^{i}=8,8.2,8.4,9$ для прямых 1,2, 3, 4 соответственно. С учетом фактора идеальности $m_{2}^{i}$ характеристическая энергия $E_{0}^{*}=m_{2}^{i} E_{0}$, где $E_{0} \cong 0.025$ эВ (см. [3]) - энергетическая характеристика материала. Для полевой эмиссии предэкспоненциальный множитель $I_{p}^{x}=0.065,1.4,4.4,9\left(10^{-5} \mathrm{~A} / \mathrm{cm}^{2}\right)$ для прямых 8, 7, 6, 5 соответственно. Фактор идеальности около $q U$ составляет $n_{1}=3$ и около $\Phi_{\text {В фактор }}$ идеальности $m_{1}^{i}=4.5,5.1,5.1,5$. Параметр $E_{00}^{*}=m_{1}^{i} E_{00}$, где $E_{00} \cong 0.020$ эВ. В случае термоэлектронной эмиссии $(s s)$ для предэкспоненциального множителя получаем: $I_{s s}^{x}=28,15,3,0.04\left(10^{-6} \mathrm{~A} / \mathrm{cm}^{2}\right)$ для прямых 9, 10, 11, 12. При этом факторы идеальности $n=1.5$ и $m=1$.

\section{4. Заключение}

Полученные в ходе расчетов значения напряжений перехода от полевой к термополевой эмиссии $U_{T h e}=2.10$, $2.35,2.60,2.78$ согласуются с данными опытов [4] $U_{E x p}=2.1,2.4,2.6,2.8$ соответственно для температур $T_{i}=77,273,361,418 \mathrm{~K}$, как показано на рис. 2. По данным этого рисунка определен температурный предэкспоненциальный множитель $T_{i}^{2}$. Большие значения факторов идеальности $n_{1}=3$ для случая полевой эмиссии и $n_{2}=5$ для термополевой эмиссии тем не менее не превосходят значения $n=6$, например, для $\mathrm{GaN} / \mathrm{GaInN}$-диодов [9]. Основное предположение настоящей работы состоит в том, что при высокой плотности поверхностных состояний дефектов в зоне контакта формируется резонансный квазиуровень Ферми. Как следствие, в диодах на основе карбида кремния и его твердых растворов потенциальный барьер на гетеропереходе имеет значение порядка высоты барьера Шоттки для соответствующего контакта металл-полупроводник $\left(\Phi_{g} \approx \Phi_{\mathrm{B}}\right)$. Ясно, что высота потенциального барьера зависит не только от значений предэкспоненциального множителя $I_{s s}, I_{p}$ и $I_{t p}$ с факторами идеальности $\left(m, m_{1}^{i}, m_{2}^{i}\right)$ и от характеристических энергий $E_{00}$ и $E_{0}$. Характер же поведения $I=I(U, x)(\mathrm{BAX})$ существенно зависит от факторов идеальности $\left(n, n_{1}, n_{2}\right)$ для $(s s)$, $(p)$ и $(t p)$ эмиссий соответственно. В итоге показано, что вольт-амперные характеристики прямых токов диодов на основе твердых растворов $\mathrm{SiC}$, рассчитанные в составной-аддитивной (4)-(6) модели, хорошо согласуются с данными экспериментов.

\section{Список литературы}

[1] С.Ю. Давыдов, А.А. Лебедев, С.К. Тихонов. ФТП, 31, 597 (1997).

[2] С.Ю. Давыдов, А.А. Лебедев, О.В. Посредник, Ю.М. Таиров. ФТП, 35, 1437 (2001).

[3] А.И. Лебедев. Физика полупроводниковых приборов (М., Физматлит, 2008).

[4] Н.П. Исмаилова, Н.В. Офицерова, Г.К Сафаралиев. Мониторинг. Наука и технологии, 1, 117 (2009).

[5] Г.К. Сафаралиев. Твердые растворы на основе карбида кремния (М., Физматлит, 2011). 
[6] В.И. Алтухов, А.В. Санкин, М.Н. Дядюк, К.С. Касьяненко, О.А. Митюгова, С.В. Филипова. Обозрение прикладной и промышленной математики, 19 (3), 423 (2012).

[7] В.И. Алтухов, И.С. Касьяненко, А.В. Санкин, Б.А. Билалов, А.С. Сигов. ФТП, 50 (9), 1190 (2015).

[8] Г.К. Сафаралиев, Н.К. Каргин, М.К. Курбанов, Б.А.Билалов, Ш.М. Рамазанов, А.С. Гусев. Вест. Нац. исслед. ядерного ун-та „МИФИ“, 3 (1), 63 (2014).

[9] Ф.Е. Шуберт. Светодиоды (М., Физматлит, 2008).

Редактор Г.А. Оганесян

\section{Nonlinear by concentration model of surface state of the Schottky barrier and calculation of current-voltage characteristics of SiC-based diodes and its solid solutions in composite model of transfer electric current}

V.I. Altukhov ${ }^{1}$, A.V. Sankin ${ }^{1}$, A.S. Sigov', D.K. Sysoev ${ }^{1}$, E.G. Yanukyan ${ }^{1}$, S.V. Filippova ${ }^{3}$

${ }^{1}$ North-Caucasian Federal University, 355009 Stavropol, Russia

${ }^{2}$ Moscow Institute of Radio Electronics and Automatics, 119454 Moscow, Russia

${ }^{3}$ Pjatigorskij medico-pharmaceutical Institute, 357500 Pyatigorsk, Russia

Abstract Proposed modified nonlinear surface by concentration model of surface states of Schottky barrier model containing local quasi-level Fermi at the border section caused by excessive surface charge. Such approach allows to explain the observed similarity of current-voltage characteristics diodes Schottky barrier $\mathrm{M} /(\mathrm{SiC})_{1-x}(\mathrm{AlN})_{x}$ and on the hetero-transitions basis of $\mathrm{SiC}$ and its solid solutions, taking into account the $\Phi_{g} \approx \Phi_{\mathrm{B}}$. The results of calculations of height Schottky barriers are consistent with those obtained by the photocurrent experiments for metals (M): Al, Ti, Cr, Ni. Current-voltage characteristics in composite-additive of transfer electric current model consistent with experiments for systems of $n-\mathrm{Me} / p-(\mathrm{SiC})_{1-x}(\mathrm{AlN})_{x}$ and $n-6 H-\mathrm{SiC} / p-(\mathrm{SiC})_{0.85}(\mathrm{AlN})_{0.15}$. 